\title{
Cohesion proteins are present in centromere protein bodies associated with avian lampbrush chromosomes
}

\author{
Alla Krasikova ${ }^{1}$, Jose Luis Barbero ${ }^{2}$ \& Elena Gaginskaya ${ }^{1 *}$ \\ ${ }^{1}$ Biological Research Institute, Saint-Petersburg State University, Oranienbaumskoie sch. 2, Stary Peterhof, \\ Saint-Petersburg 198504, Russia; Tel: (+7-812) 427-7311; Fax: (+7-812) 427-7310; \\ E-mail: chromas@paloma.spbu.ru; ${ }^{2}$ Department of Immunology and Oncology,Centro Nacional \\ de Biotecnologia, UAM Campus de Cantoblanco, Madrid E-28049, Spain \\ * Correspondence
}

Received 23 July 2005. Received in revised form and accepted for publication by Herbert Macgregor 22 August 2005

Key words: avian oocyte, centromere, cohesin, lampbrush chromosome, protein bodies

\begin{abstract}
Proteins of sister chromatid cohesion are important for maintenance of meiotic chromosome structure and retention of homologous chromosomes in bivalents during diplotene. Localization of the cohesion proteins within nuclei of growing oocytes merits special attention, particularly in avian oocytes, in which diplotene chromosomes assume the form of lampbrush chromosomes (LBCs). We performed indirect immunostaining using antibodies against cohesins $\mathrm{SMC} 1 \alpha, \mathrm{SMC} 1 \beta, \mathrm{SMC} 3, \mathrm{Rad} 21$, and the SA/STAG family on chaffinch, pigeon and duck LBCs spreads, and frozen ovary sections. On LBC spreads, antibodies to the majority of cohesins showed punctate staining on chromosome axes. LBC lateral loops, where sister chromatids are separated, did not show cohesin components. The spherical entities attached to the LBC centromeres in avian germinal vesicles, the so-called protein bodies (PBs), were enriched in SMC1 $\alpha$, SMC3, Rad21, STAG1 and STAG2. The synaptonemal complex component SYCP3, which also participates in cohesion, was detected in the axes of avian lampbrush bivalents and, to a greater degree, in the PBs. In vitellogenic oocytes, cohesion proteins persist in the PBs associated with condensing bivalents when they concentrate into the karyosphere. These results indicate that cohesion proteins accumulate in centromere PBs in avian oocytes and are involved in structural maintenance of lampbrush chromosome axes.
\end{abstract}

\section{Introduction}

In most avian species studied, the nucleus of the growing diplotene oocyte, or germinal vesicle $(\mathrm{GV})$, shows nearly 80 conspicuous round structures termed protein bodies (PBs) at chromosome centromeric regions (Gaginskaya \& Gruzova 1969, Gaginskaya 1972, Solovei et al. 1996, Saifitdinova et al. 2003). Diplotene bivalents of growing avian oocytes assume the form of giant lampbrush chromosomes (LBCs) consisting of two homologues united by chiasmata.
Each chromosome in the lampbrush bivalent appears as a set of chromomeres (compacted chromatin), interchromomere regions, and pairs of lateral loops in which active transcription takes place (reviewed by Callan 1986, Morgan 2002); these features are usually identical in the two half-bivalents. In pigeon and chaffinch GVs, PBs form adjacent to the chromatin blocks of centromeric satellite repeats PR1 and FCP, respectively (Solovei et al. 1996, Saifitdinova et al. 2003). The PBs do not contain splicing factors, splicing RNA or transcribing RNA polymerase II (Saifitdinova et al. 
2003, Krasikova et al. 2004), and thus differ from Cajal bodies and B-snurposomes (or speckles). The role of the PBs remains unclear, but it seems that these structures reflect a thus-far unknown centromere function. As oocytes grow, the PBs vacuolize, increase in size and fuse to each other during karyosphere formation, with the chromosomes attached to them. This process correlates with condensation of the bivalents within the GV (Gaginskaya 1972).

The only PB component reported to date is DNA topoisomerase II (topo II; Krasikova et al. 2004), which is known to be involved in topological organization of chromatin (Earnshaw et al. 1985). We thus hypothesized that PBs may contain other proteins implicated in structural maintenance of chromosomes and important for centromere function. Candidate proteins would include the cohesins, which are key molecules in the cohesion of sister chromatids at chromosome arms and centromeric regions.

Components of multiprotein cohesin complexes participate in sister chromatid union, chromosome attachment to the spindle, and in DNA repair (reviewed by Morrison et al. 2003). Sister chromatid cohesion is required for correct chromosome segregation during cell division, so that daughter cells receive identical chromosome sets. The cohesion mechanism is still unclear (reviewed by Haering \& Nasmyth 2003). In the 'ring' model, two cohesins of the structural maintenance of chromosome family (SMC1 $\alpha$ and SMC3) form an unclosed heterodimeric ring embracing two sister chromatids. The Rad 21 subunit binds to the open ends of the SMC1-SMC3 heterodimer, closing the ring structure (Gruber et al. 2003); Rad21 was thus termed a 'kleisin' (Schleiffer et al. 2003). A STAG/SA (stromal antigen) protein (STAG1 or STAG2 in somatic cells and STAG3 in germ line cells) also participates in the formation of the four-subunit cohesin complexes (Losada et al. 2000, Pezzi et al. 2000, Prieto et al. 2001).

Cohesion of sister chromatids during meiosis has specific features. In mammals, chromosome arm cohesion is lost before centromere cohesion during the two meiotic divisions; only centromeric regions remain paired from anaphase I to anaphase II (reviewed by Cohen-Fix 2000, Jessberger 2002). Rec8, STAG3 and SMC1 $\beta$ are the respective meiosisspecific isoforms of the cohesin subunits, $\operatorname{Rad} 21$, STAG and SMC1 $\alpha$ (Watanabe \& Nurse 1999, Prieto et al. 2001, Revenkova et al. 2001); nevertheless, mitotic cohesin subunits have also been found in meiosis (Prieto et al. 2002, 2004, Parra et al. 2004, $\mathrm{Xu}$ et al. 2004). During meiotic prophase I, homologous chromosomes pair to form the synaptonemal complex (SC), whose formation is dependent on the cohesin axis. Lateral elements of the SC, formed mainly by SYCP2/SCP2 and SYCP3/SCP3 proteins, are linked by the central element. It was suggested that SMC1 and SMC3 bind directly to the synaptonemal complex-specific proteins SYCP2 and SYCP3 (Eijpe et al. 2000). Condensation and cohesion of meiotic chromatids in the lampbrush bivalents, a characteristic of middle and later diplotene stages in avian oogenesis, has yet to be studied in detail.

Due to their enormous size, LBCs permit easy visualization of chromosome regions in different states of chromatin compaction and chromatid cohesion (Macgregor et al. 1997). Interchromomeric segments of LBC axes appear to be the areas of close junction of sister chromatids, while pairs of lateral loops represent regions at which sister chromatids are separated symmetrically. The chromomere is thought to be a chromosome region in which high chromatin condensation levels persist, in contrast to the lateral loops. Nonetheless, chromatid organization within the chromomere and the implication of cohesin complex proteins in the structural maintenance of LBCs has yet to be studied.

Here we report the localization of cohesin complex proteins and SC components in LBCs and in associated PBs at several stages of avian oocyte growth. We also discuss the contribution of cohesion proteins to the organization of specific intranuclear structures in the oocyte nucleus.

\section{Materials and methods}

\section{Lampbrush chromosome preparation}

Chaffinch (Fringilla coelebs), pigeon (Columba livia) and duck (Anas platyrhynchus) LBCs were isolated manually from oocyte GVs according to standard techniques (Solovei et al. 1993, 1994, Saifitdinova et al. 2003). $\mathrm{MgCl}_{2}$ was added to a final concentration of $1 \mathrm{mmol} / \mathrm{L}$ to the chromosome isolation and fixing solutions. After brief fixing in $2 \%$ paraformaldehyde, preparations were post-fixed in $70 \%$ methanol overnight. Preparations were not dried before immunostaining. 


\section{Oocyte sectioning}

Dissected ovaries were fixed in $4 \%$ paraformaldehyde $(2 \mathrm{~h}$, room temperature (RT)) and washed in PBS. Fixed tissue was frozen in liquid nitrogen and cryotome sections $(20 \mu \mathrm{m})$ were obtained. Frozen sections were placed on glass slides treated with 3 -aminopropyltriethoxysilane, then fixed in $4 \%$ paraformaldehyde (10 min). Preparations were washed in PBS and used for immunofluorescence staining.

\section{Antibodies and immunofluorescence staining}

For immunostaining of avian LBCs and ovary sections, we used the following rabbit polyclonal antibodies: K988 anti-human SMC1 $\alpha$, K974 anti-mouse

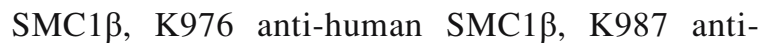
human SMC3, K853 and K854 anti-human $\operatorname{Rad} 21$, K923 anti-human STAG1, K828 anti-human STAG2, K919 anti-rat SYCP1, K921, K1034 and K1037 antirat SYCP3 (Table 1, Prieto et al. 2004), and mouse monoclonal antibody (mAb) 4A6 anti-amphibian topo II (Hock et al. 1996). NCBI BLAST was used to search for certain epitopes in the amino acid sequences of chicken proteins or predicted proteins (http://www.ncbi.nlm.nih.gov; see Table 1).

LBC spreads were blocked in PBS containing $1 \%$ blocking reagent (Roche; $1 \mathrm{~h}, \mathrm{RT}$ ), then incubated with primary antibody ( $1 \mathrm{~h}, \mathrm{RT}$; dilutions as recommended by authors). Slides were washed in PBS, $0.05 \%$ Tween-20 and incubated (1 h, RT) in Cy3-goat anti-mouse $\mathrm{IgG}+\mathrm{IgM}$ antibody (Jackson ImmunoResearch) or Cy3-conjugated goat anti-rabbit, alone or with goat Alexa-488-anti-mouse antibody (Molecular Probes). Slides were washed in PBS, $0.05 \%$ Tween-20 and mounted in DABCO antifade solution containing $1 \mu \mathrm{g} / \mathrm{ml}$ DAPI.

\section{Fluorescence microscopy}

Preparations were examined on a DMRXA fluorescence microscope (Leica Microsystems Wetzlar $\mathrm{GmbH}$, Germany) equipped with a Fluotar 100/ 1.30 NA objective, appropriate filters, and a blackand-white CCD camera (Cohu). QFISH software (Leica Cambridge Ltd.) was used to acquire and process multicolour microscopic images. Confocal laser scanning microscopy was carried out on a Leica TCS SL microscope equipped with $\mathrm{HeNe}(543 \mathrm{~nm})$ and $\mathrm{Ar}$ $(488 \mathrm{~nm})$ lasers.

\section{Protein preparation and Western blotting}

Chaffinch GVs were isolated manually and collected in ' $5: 1$ ' isolation buffer $(83 \mathrm{mmol} / \mathrm{L} \mathrm{KCl}, 17 \mathrm{mmol} / \mathrm{L}$ $\mathrm{NaCl}, 6.5 \mathrm{mmol} / \mathrm{L} \mathrm{Na} \mathrm{HPO}_{4}, 3.5 \mathrm{mmol} / \mathrm{L} \mathrm{KH}_{2} \mathrm{PO}_{4}$, $1 \mathrm{mmol} / \mathrm{L} \mathrm{MgCl}_{2}, 1 \mathrm{mmol} / \mathrm{L}$ dithiothreitol (DDT), $\mathrm{pH}$ 7.0) with complete protease inhibitor cocktail (Roche). The GV membranes were mechanically disrupted and then the proteins were dissolved in SDS sample buffer. Nuclei from pigeon testis cells were isolated as described by Berezney \& Coffey (1974). Purified testis nuclei were extracted $\left(1 \mathrm{~h}, 4^{\circ} \mathrm{C}\right)$ in $10 \mathrm{mmol} / \mathrm{L}$ Tris- $\mathrm{HCl}, \mathrm{pH} 8.0,0.1 \mathrm{mmol} / \mathrm{L} \mathrm{MgCl}_{2}$, $0.2 \mathrm{mmol} / \mathrm{L}$ PMSF, $0.5 \mathrm{mmol} / \mathrm{L}$ dithiothreitol, $5 \%$ glycerol and $0.35 \mathrm{~mol} / \mathrm{L} \mathrm{NaCl}$, centrifuged $(10 \mathrm{~min}$, $5000 \mathrm{~g}$ ), and the supernatant nuclear extracts collected. Proteins from chaffinch oocyte GVs and from pigeon testis cell nuclear extracts were separated on $10 \%$ polyacrylamide/SDS gels, and transferred to Hybond $\mathrm{C}+$ (Amersham, UK) using a minitransfer cell (BioRad, Hercules CA). Alternatively, gels were fixed in 50\% ethanol and silver stained (Dunn 1993). Membranes were blocked in 5\% fat-free dry milk in TBS, washed in TBS, $0.1 \%$ Tween-20 and incubated with primary antibody $(1 \mathrm{~h}, \mathrm{RT}, 1: 100$ dilution in blocking solution). After three washes in TBS, $0.1 \%$ Tween20, the membrane was incubated with AP-conjugated goat anti-rabbit antibody (Santa Cruz, 1:100 00). After washing, bound antibody was visualized using BCIP/ NBT (Sigma).

\section{Results}

\section{Testing for antibody specificity to avian} cohesion proteins

Antibodies used in this study were initially raised against peptides of human and/or mouse cohesion proteins and rat SC components (Prieto et al. 2004). To determine the suitability of these sera for the study of avian oocytes, we searched for these peptides in the chicken protein databases; the corresponding peptides in chicken cohesins and SC lateral element component SYCP3 are shown in Table 1. We predicted that the majority of the polyclonal sera would recognize the avian proteins as these epitopes are highly conserved. Western blot analysis of protein extracts from pigeon testis nuclei using anti-SMC1 $\alpha,-S M C 1 \beta,-S M C 3,-\operatorname{Rad} 21,-S T A G 1$, 


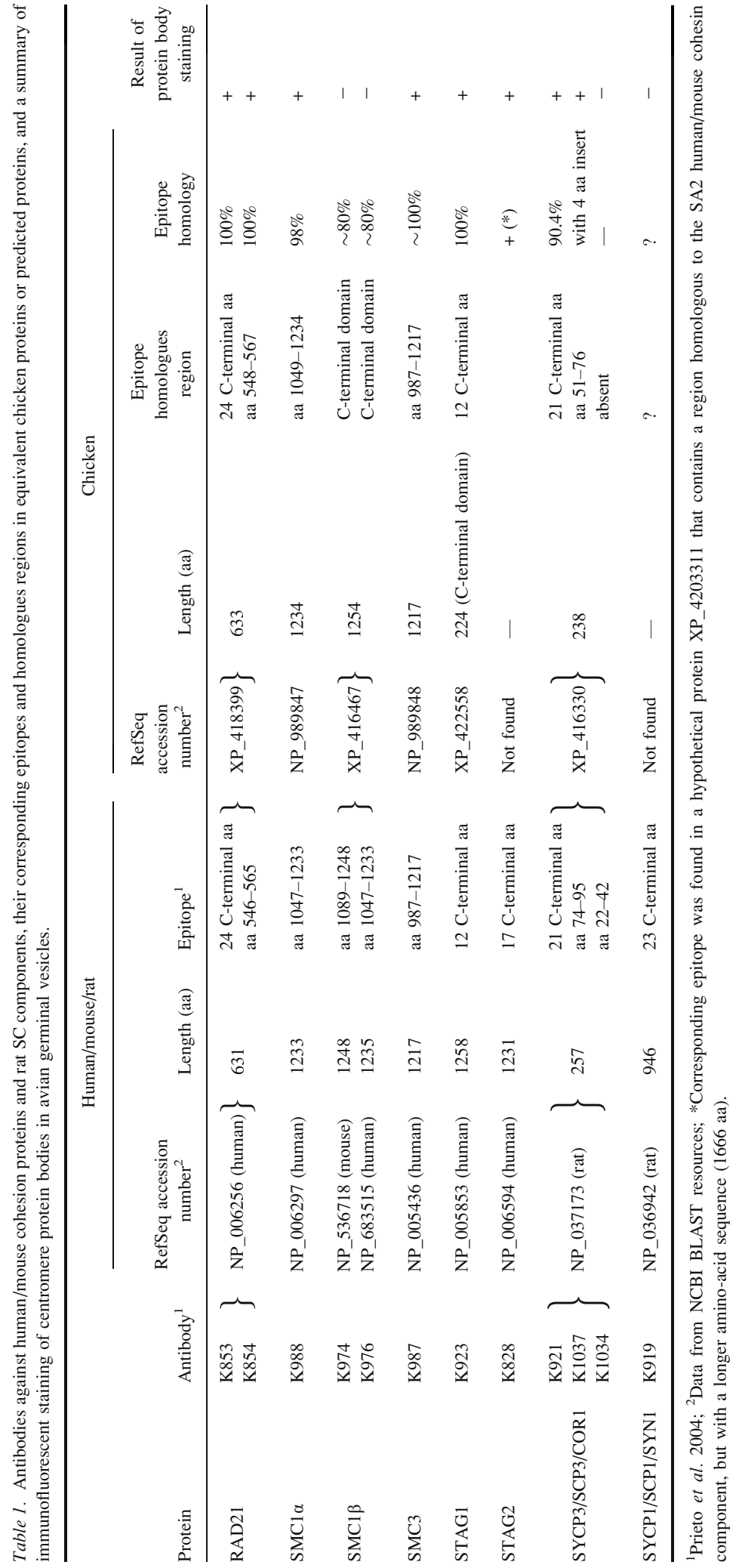


-STAG2, -SYCP1 and -SYCP3 antibodies specifically detected bands of appropriate mobility (Figure 1a). In Western blot and immunofluorescence experiments, non-immune rabbit serum was used at the same dilution as antibodies as a negative control; no signal was detected (not shown). STAG1 and STAG2 proteins were observed as doublets, which may correspond to distinct phosphorylation states, as STAG2 phosphorylation is necessary for cohesin dissociation from the chromosome arms (Hauf et al. 2005). Two antibodies against different $\operatorname{Rad} 21$ epitopes detected several bands in pigeon testis nuclear extracts; the major band corresponds to the uncleaved protein and the weaker bands probably represent breakdown products. Two distinct antibodies identified SYCP3 as a doublet, concurring with data for the rat COR1/ SCP3 homologue (Dobson et al. 1994).

To study cohesion proteins in avian diplotene oocyte nuclei, we analysed GV contents by Western blot (Figure 1b). Immunoblotting with anti-Rad21 antibody showed that this kleisin component of the cohesin complex is found in the GV; no cleavage products were observed. STAG2 migrated as a doublet, as occurs in testis nuclear extracts; SYCP3 was detected as a single band, in contrast to testis nuclear proteins.

\section{Cohesin component distribution on avian lampbrush chromosomes and associated structures}

To analyse cohesin localization on lampbrush chromosomes and in associated structures, GVs spreads of pigeon, chaffinch and duck oocytes were labelled with antibodies to cohesin axis components ( $\mathrm{SMC} 1 \alpha$, SMC1 $\beta$, SMC3) and examined by immunofluorescence. Antibodies to SMC1 $\alpha$ and SMC3 bound predominantly to $\mathrm{PBs}$ associated with LBC centromeres (Figure 2a, b). We also found moderate scattered staining of both half-bivalents at symmetrical positions. In the $\mathrm{ZW}$ sex bivalent, in which non-homologous chromosomes are coupled by a single chiasma, chromosome axis staining was not symmetrical (Figure 2a, $a^{\prime \prime}$ ). Maximum cohesin staining was observed in interchromomere segments of chromosomes, especially in chromosome regions in which the number of loops (i.e. unpaired regions) or their overall lengths were at a minimum (i.e. some microchromosomes and W-chromosome) (Figure 2). SMC protein staining was not observed in the lateral loops. Chromomeres, the DAPI-stained regions of compacted a

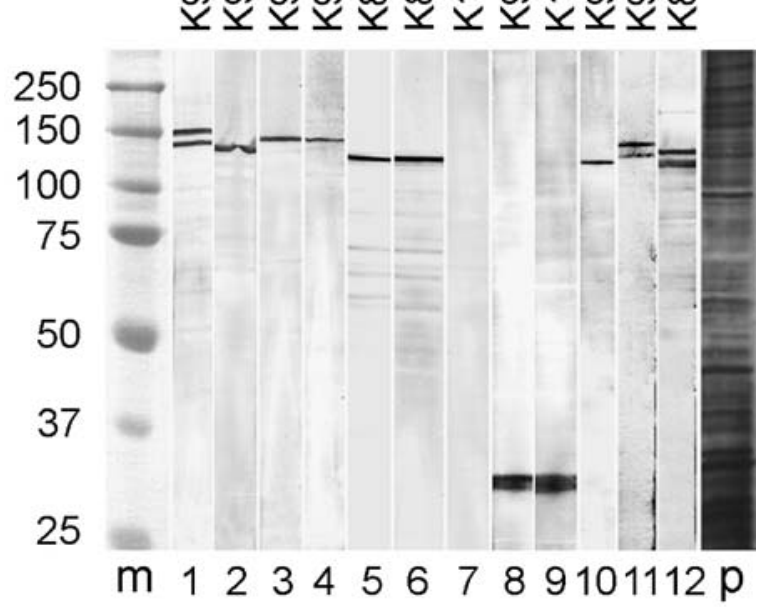

b

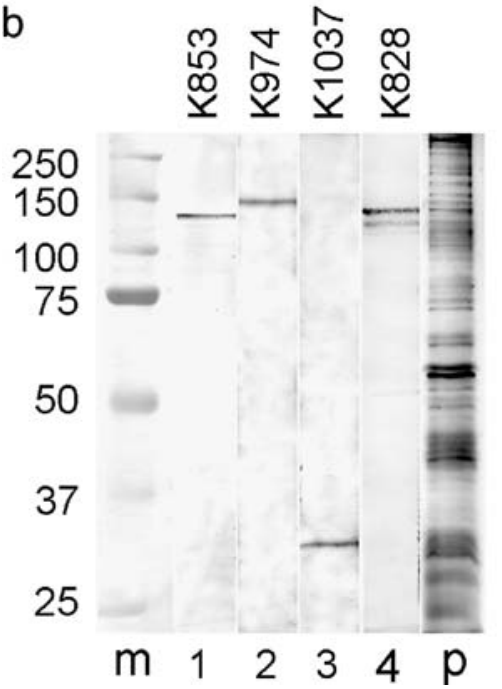

Figure 1. Western blotting analysis of the sister chromatid cohesion proteins from avian germ cells. (a) Analysis of rabbit anti-cohesin and -SYCP3 antibodies for specificity to avian proteins. Nuclear proteins from pigeon testis cells were separated in 10\% SDS-PAGE. Lanes 1-12, SMC1 $\alpha$ (antibody K988), SMC3 (K987), SMC1ß (K974 and K976), Rad21 (K854 and K853), SYCP3 (K1034, K921 and K1037), SYCP1 (K919), STAG1 (K923) and STAG2 (K828), respectively. Molecular masses of protein standards $(\mathrm{m})$ are indicated in $\mathrm{kDa}$; $\mathrm{p}$ is the staining of total nuclear proteins from pigeon testis cells. (b) Detection of cohesion proteins in chaffinch GVs. Lanes 1-4, staining with rabbit antiRad21 (K853), SMC1 $\beta$ (K974), SYCP3 (K1037), and STAG2 (K828), respectively. Each lane contains proteins from 25 manually isolated GVs. Molecular masses of protein standards (m) are indicated in $\mathrm{kDa} ; \mathrm{p}$ is the staining of total proteins from 35 chaffinch GVs. 
chromatin, were either unlabelled or were not completely covered with SMC1 and SMC3 proteins; in the latter case, only chromomere cores showed detectable amounts of these cohesin components.

In Western blots, two different anti-SMC1 $\beta$ antibodies recognized bands of appropriate $M_{r}$ in chaffinch GVs and pigeon testis nuclear extracts (Figure 1), whereas SMC1 $\beta$ was found neither on LBCs nor in associated PBs in immunofluorescence studies (not shown). As the SMC1 $\beta$ epitopes to which antibodies were raised are not identical for avian, human and murine proteins (Table 1), conformational differences may hinder antibody recognition.

We examined the distribution of other cohesin components $(\operatorname{Rad} 21$, STAG1 and STAG2; Figure 2c-g), and observed patterns similar to those for SMC3 and SMC1 $\alpha$. The signal of all antibodies against these proteins was detected along the chromosome axes in a punctate pattern (Figure 2d, $\mathrm{d}^{\prime \prime}$ ). PBs on LBCs showed intense fluorescence with antibodies against $\operatorname{Rad} 21$, STAG1 and STAG2 proteins (Figure 2c-g). The two anti-Rad21 antibodies (K853 and K854) produced similar LBC and PB labelling patterns.

We analysed the localization of SYCP1 and SYCP3, which are components of the central and lateral elements of the SC respectively, on chaffinch and pigeon LBCs spreads. SYCP1 was not detected in either LBCs or PBs in these preparations (not shown). The signal of two different anti-SYCP3 antibodies was strong in centromere-associated PBs, similar to anti-cohesin labelling (Figure 2h, i). SYCP3 is involved in mammalian sister chromatid cohesion during meiosis I; bright SYCP3 labelling of the axes in both half-bivalents coincided with the localization of cohesin components (Figure $2 \mathrm{i}, \mathrm{i}^{\prime \prime}$ ), suggesting that SYCP3 has a similar role in the avian model.

In addition to the centromere PBs, a number of PBs form occasionally on the LBC axis. For chaffinch LBCs, these facultative $\mathrm{PBs}$ are associated with interstitial sites of the centromeric tandem FCP repeat (Saifitdinova et al. 2003), and show cohesins and SYCP3, similar to the centromere PBs (Figure 2h, i). Rare small spherules on the PB surface appear to have a protein composition that differs from that of PBs itself; most cohesin components studied were not found in the spherules in chaffinch GVs (Figure 2f, $\mathrm{f}^{\prime}$ ).

In the early lampbrush phase, no prominent spherical structures (i.e. PBs) are observed in association with the centromeric regions of bivalents. STAG2 labelling was found in the axes of the homologous chromosomes dissected from these oocytes. Notably, brighter signals were observed at the putative centromeric regions, determined by centromeric indexes and marker loops/chromomeres location, on both microand macrochromosomes (Figure $2 \mathrm{j}, \mathrm{j}^{\prime}$ ) at sites of future PB formation. Prominent STAG2 agglomerates were also found to occur in the GV nucleoplasm (Figure $2 \mathrm{j}, \mathrm{j}^{\prime}$ ).

Confocal microscopy analysis of cohesin components and DNA topoisomerase II localization within protein bodies

In chaffinch, pigeon and duck oocytes, we recently demonstrated that DNA topoisomerase II (topo II) is located predominantly in the dense component of PBs but not in the vacuoles, as shown by immunoelectron microscopy (Krasikova et al. 2004). PB size (up to $12 \mu \mathrm{m}$ diameter) allows analysis of protein distribution and comparative localization by confocal microscopy. Here we performed indirect double immunostaining of $\mathrm{PBs}$ on $\mathrm{GV}$ spreads using the 4A6 anti-amphibian topo II mAb (Hock et al. 1996) and an anti-Rad21 (K854) or anti-SMC3 antibody. These proteins were non-uniformly distributed within PBs, cohesins being localized in the same PB component as topo II (Figure 3a). This part of the PB shows a denser texture in electron microscopy (Tsvetkov \& Gaginskaya 1983). Notably, DNA topo II and $\operatorname{Rad} 21$ do not entirely co-localize within this dense PB component.

\section{Cohesin localization on cryosections of adult bird ovaries}

Because most nuclear proteins that are not associated with LBCs and other nuclear structures are washed out during pre- and post-fixative treatments, data

Figure 2. Immunofluorescence detection of sister chromatid cohesion proteins on chaffinch, Fringilla coelebs (a, c-j) and pigeon, Columba livia (b) lampbrush chromosomes (LBCs). (a-j) Immunostaining with rabbit anti-SMC3 (K987), SMC1 $\alpha$ (K988), STAG1 (K923), STAG2 (K828), Rad21 (K853) and SYCP3 (K1037) antibodies. Centromere protein bodies and LBC axes show punctate staining (red). Chromosomes are counterstained with DAPI (blue). $\left(\mathbf{a}^{\prime}-\mathbf{j}^{\prime}\right)$ Phase contrast. $\left(\mathbf{a}^{\prime \prime}, \mathbf{d}^{\prime \prime}, \mathbf{i}^{\prime \prime}\right)$ Only immunostaining is shown (without DAPI). Arrows indicate centromere protein bodies in $\mathbf{a}-\mathbf{i}$ and putative centromeric regions in $\mathbf{j}$; $\mathbf{w}, \mathrm{W}$ chromosome in $\mathrm{ZW}$ bivalent; s, spherule on the protein body surface; arrowheads, facultative protein bodies; a, STAG2 agglomerates. Scale bars $=10 \mu \mathrm{m}$. 


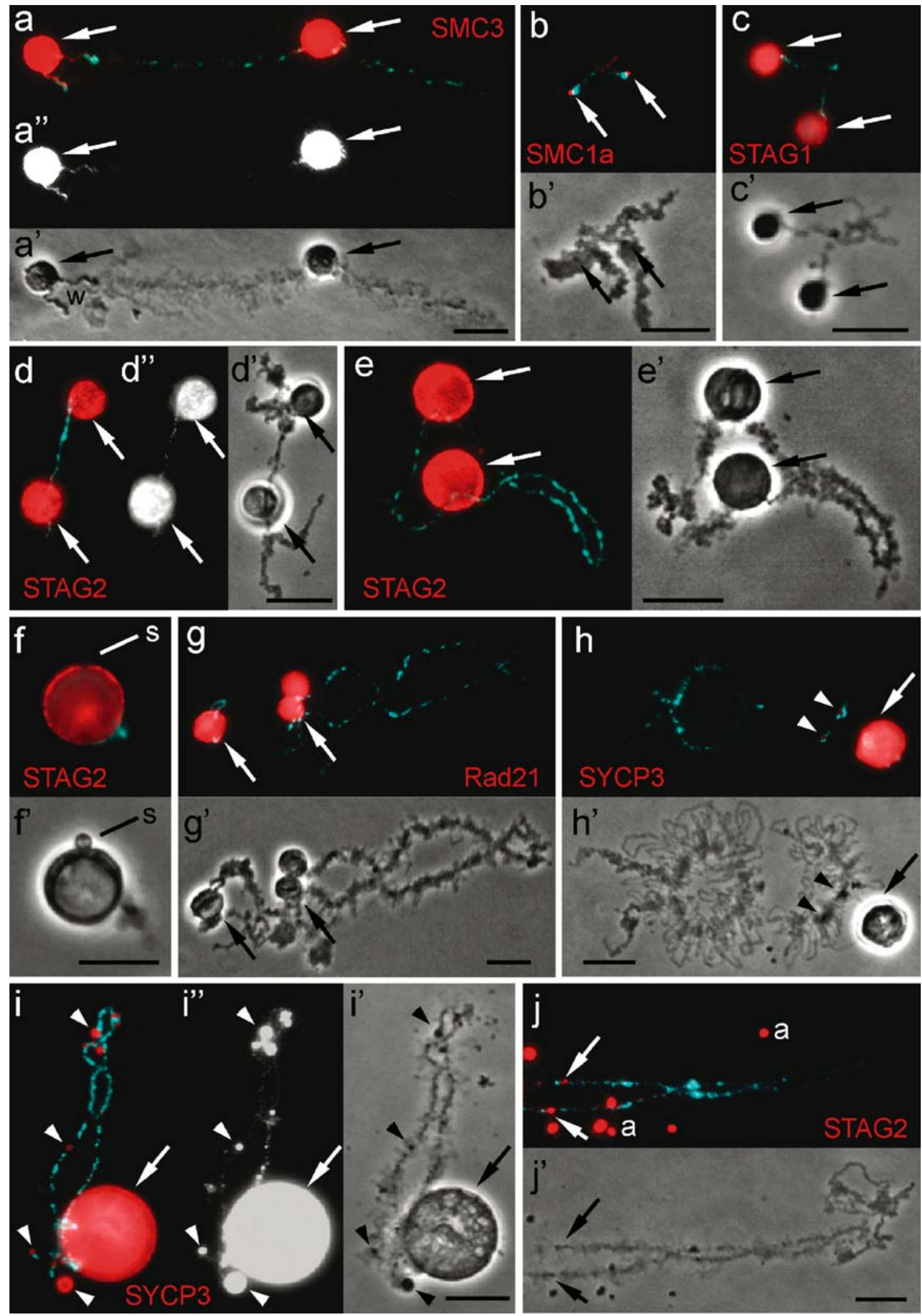



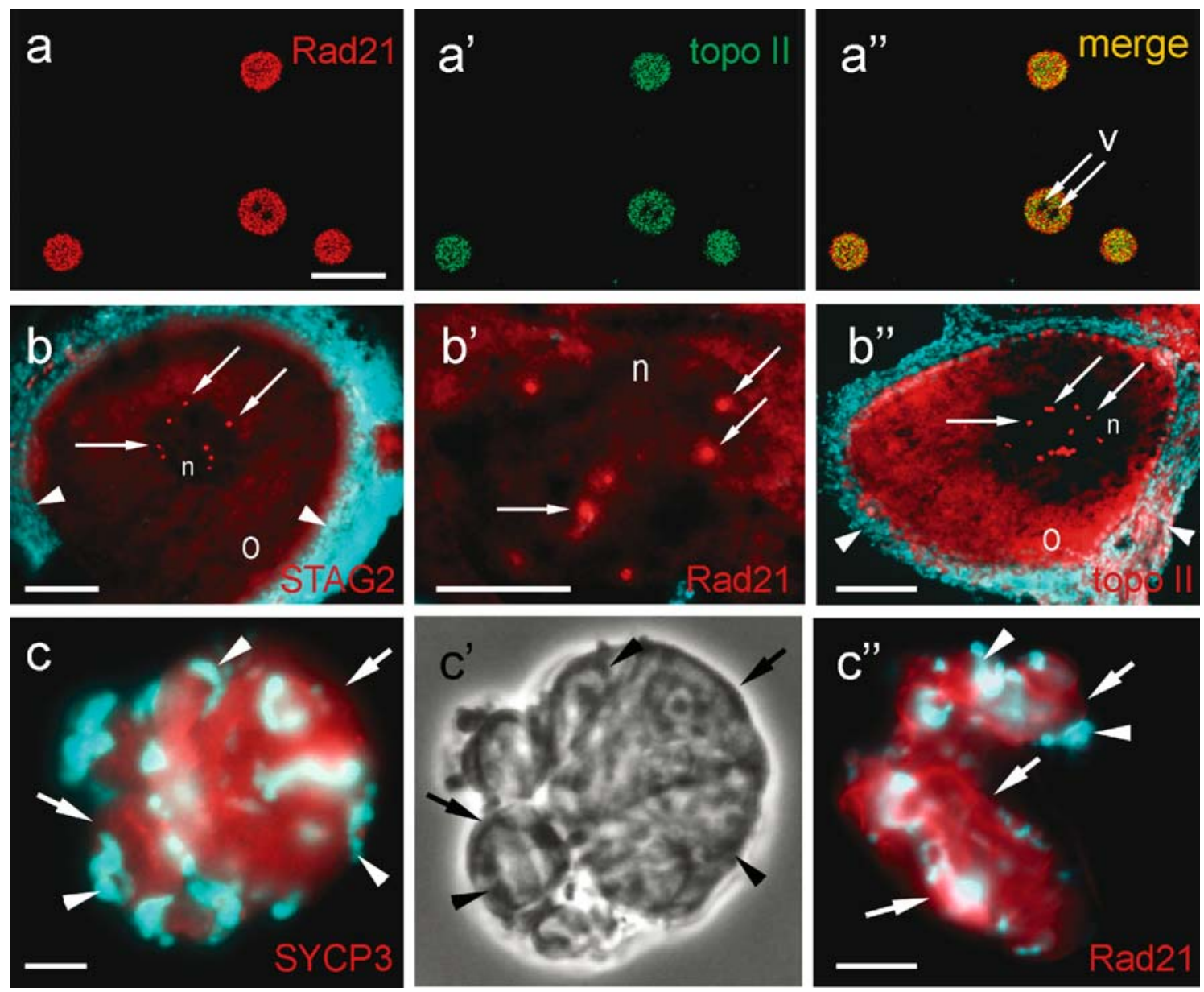

Figure 3. Immunofluorescence localization of cohesion proteins in avian centromere protein bodies and karyospheres. (a- $\left.\mathbf{a}^{\prime \prime}\right)$ Comparative localization of cohesin subunit Rad21 and DNA topoisomerase II (topo II) within centromere protein bodies. Representative confocal sections through four protein bodies from a duck GV, immunostained with anti-Rad21 antibody (K854) (red, a) and anti-topo II (4A6 mAb) (green, $\left.\mathbf{a}^{\prime}\right)$. $\left(\mathbf{a}^{\prime \prime}\right)$ Merge image. Note that vacuoles (v) in protein bodies are not stained. Scale bar $=16 \mu \mathrm{m}$. (b- $\left.\mathbf{b}^{\prime \prime}\right)$ Distribution of cohesins and topo II within pigeon $\left(\mathbf{b}, \mathbf{b}^{\prime}\right)$ and chaffinch $\left(\mathbf{b}^{\prime \prime}\right)$ oocytes. Immunofluorescence staining of frozen ovary sections with anti-STAG2 (K828) (b), -Rad21

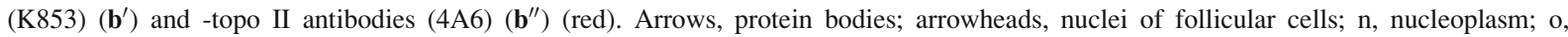
ooplasm. DNA is counterstained with DAPI (blue). Scale bars $=100 \mu \mathrm{m}$ in $\mathbf{b}, \mathbf{b}^{\prime \prime}$ and $=50 \mu \mathrm{m}$ in $\mathbf{b}^{\prime}$. (c- $-\mathbf{c}^{\prime \prime}$ ) Karyospheres from chaffinch

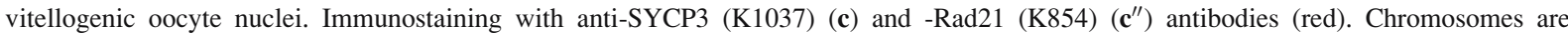
counterstained with DAPI (blue). ( $\left.\mathbf{c}^{\prime}\right)$ Phase contrast image of the karyosphere shown on panel c. Arrows indicate fusing centromere protein bodies; arrowheads show condensing bivalents. Scale bars $=20 \mu \mathrm{m}$.

from GV spreads do not correctly compare protein concentrations within intranuclear structures and the nucleoplasm (Doyle et al. 2002). To determine cohesin distribution throughout the oocyte, including the nucleus, we immunostained pigeon and chaffinch ovary cryosections with anti-STAG2 and anti-Rad21 antibodies. Generalized STAG2 and Rad21 signals were found in oocyte cytoplasm at all stages, and the nucleoplasm was less intensely labelled. In GV of larger oocytes, multiple round structures gave a strong positive reaction against a background of poorly-labelled nucleoplasm (Figure $3 b, b^{\prime}$ ). These structures correspond to $\mathrm{PBs}$, as determined from phase-contrast images (not shown). Similar staining was observed for 4A6 anti-topo II mAb on frozen sections (Figure $3 b^{\prime \prime}$ ), concurring with immunogold labelling of avian GV thin sections (Krasikova et al. 2004). 
Cohesion protein distribution within the karyosphere in chaffinch germinal vesicles

During chromosome condensation, cohesion complexes are replaced by condensation proteins (condensins), except in centromeric regions and some discrete sites on chromosome arms (reviewed by Hagstrom \& Meyer 2003). We analysed the distribution of cohesion proteins in the nucleus during later stages of chaffinch oogenesis in relation to chromosome condensation and karyosphere formation. Within the karyosphere, fluorescence of centromere PBs persists after staining with anti-Rad21 and -SYCP3 antibodies, whereas the labelling of condensing chromosomes almost disappears (Figure 3c).

\section{Discussion}

Sister chromatid cohesion proteins in avian lampbrush chromosome axes

The chromosomal axes of LBCs are composed of discrete chromomeres containing highly compacted chromatin and interchromomeric regions of joined sister chromatids. Our results suggest that cohesion complex components act in specific LBC axis domains. Rad21, SMC3, SMC1 $\alpha$, STAG1 and STAG2 cohesins localize non-uniformly in the interchromomeric regions, showing a punctate pattern at homologous positions, possibly reflecting chromatin region-specific interaction of cohesins. Lateral loops extend symmetrically from the chromomeres as a result of transcription, representing individual sister chromatid regions that are clearly distinguished due to the polarized ribonucleoprotein (RNP) matrix on each transcription unit. Cohesins are displaced from genes when transcription is turned on, and accumulate near gene ends (Lengronne et al. 2004, Ross \& Cohen-Fix 2004). Here we show that cohesins are absent from avian LBC loop regions.

Gall et al. (2004) recently raised the question as to whether sister chromatids are paired or unassociated within LBC chromomeres. Injection of haploid sperm nuclei into GVs showed that chromomeric-loop structures can assemble from a single chromatid (Gall \& Murphy 1998); it was thus proposed that chromomere formation does not require interchromatid interactions (Morgan 2002). We localized SMC3, a common component of meiosis- and mitosis-specific cohesin complexes, on the axes of avian LBCs, showing that SMC3 does not cover the entire DAPIpositive area of the chromomere. This supports the hypothesis that chromomeric chromatin is represented by a set of small loops, the sister chromatids being separated (Kropotova \& Gaginskaya 1984). In amphibian GVs, each chromomere contains the condensin XCAP-D2; the signal was directly proportional to the amount of chromomere DNA, and was not detected in non-chromomeric regions of the LBC axis (Beenders et al. 2003). Condensins are involved in intrachromatid organization; within the chromomere, condensins might thus organize DNA of each chromatid into separate loops.

Remnants of lateral elements of the SC may remain as cohesive material between sister chromatids (Solari 1981), and are required for cohesin core maintenance at diplotene (Kouznetsova et al. 2005); our finding of SYCP3 association with the axes of avian lampbrush chromosomes concurs with these observations. Cohesin is lost during chromosome condensation in prophase of the mitotic cell cycle (reviewed by Hagstrom \& Meyer 2003). We did not detect cohesins or SYCP3 in the axes of chromosomes condensed into karyospheres in late avian $\mathrm{GVs}$, suggesting that the majority of these cohesion proteins are removed from DNA by condensin action. DNA topo II is absent from the axes of amphibian and avian lampbrush bivalents (Fisher et al. 1993, Hock et al. 1996, Krasikova et al. 2004). All together, these data indicate that the cohesin complex and SC lateral element proteins, as well as the condensins, have a key role in maintaining specific chromosome structure in the lampbrush phase.

\section{Centromere protein bodies associated with avian lampbrush chromosomes contain sister chromatid cohesion proteins}

The protein bodies at centromeric regions of meiotic diplotene bivalents in growing avian oocytes are enriched in cohesins SMC1 $\alpha$, SMC3, Rad21, STAG1 and STAG2 (Table 1, last column). The high concentration of these components within PBs in contrast to the nucleoplasm suggests that centromere $\mathrm{PBs}$ are specific nuclear domains involved in cohesin accumulation. SYCP3 was also found in centromere $\mathrm{PBs}$ in chaffinch and pigeon GVs. Similarly, substantial amounts of SYCP3, originally termed COR1, were associated with sister centromeres in metaphase 
I (Dobson et al. 1994). Another group reported SYCP3 and Rad21 release from chromosome arms and accumulation at centromere regions in mouse diplotene spermatocytes (Parra et al. 2004).

In chaffinch oocytes, we found prominent STAG2 agglomerates in the nucleoplasm at the beginning of bivalent decondensation into $\mathrm{LBCs}$, prior to $\mathrm{PB}$ formation. This observation appears analogous to results for mammalian SYCP3 and Rad21 (Parra et al. 2004) and for STAG3 (Prieto et al. 2001), which are released from chromosomes at diplotene and accumulate in the nucleoplasm as large aggregates. The significance of this event is not known.

PB size differs greatly at early stages of formation and in the later oocytes (up to 10-fold) (Gaginskaya 1972), and our results show that the cohesion protein signals within PBs increase during oocyte growth. Nonetheless, the amount of DNA involved in PB formation must be constant. The cohesion proteins inside PBs thus may have other functions in addition to sister centromere cohesion.

Active centromeric regions are highly enriched in cohesins and DNA topo II, whose binding to the centromere can be dynamic (reviewed in Meluh \& Strunnikov 2002). Firooznia et al. (2005) asked whether meiosis-specific cohesin complexes, which maintain sister centromere cohesion during meiosis, colocalize with $\operatorname{Rad} 21$ and STAG2, or whether these latter proteins are included in another 'cohesin-like entity' at the centromere region. Parra et al. recently showed that Rad21 and SYCP3 are involved in monopolar attachment of sister kinetochores during meiosis I (Parra et al. 2004). The accumulation of cohesins such as SMC1 $\alpha, \operatorname{Rad} 21$, STAG1, STAG2 and SMC3 as well as SYCP3 in avian PBs suggests additional functions unrelated to sister chromatid cohesion. Cohesins and SYCP3 persist in the PBs during karyosphere formation in later chaffinch oocytes. We propose that the PBs may serve as a cohesin and topo II storage compartment until early embryogenesis.

Structures similar to PBs, containing cohesion proteins and DNA topo II but no components of gene expression apparatus, have not been identified in oocytes of non-avian species. There is, nonetheless, a certain resemblance between $\mathrm{PBs}$ and axial granules, which form on amphibian LBC axes (Callan 1986). LBC axial granules in Pleurodeles walt can fuse with one another and contain DNA topo II (Pyne et al. 1995, Hock et al. 1996). Centromere and telomere granules on the lampbrush bivalents of Notophthalmus viridescens have neither hnRNP nor snRNP, may reach a diameter of $20 \mu \mathrm{m}$, and are proposed to belong to a more 'general class of structures' (Gall 1992). These entities, which form in the oocyte nucleus in various species, should be further compared with PBs. The features of $\mathrm{PB}$ development on avian LBCs, their structural reorganization during karyosphere formation, and their association with centromeric satellite DNA, mark them as a distinctive type of nuclear body whose functional significance should be further explored.

\section{Acknowledgements}

We thank R. Hock for mAb 4A6, I. Prieto for anticohesion protein antibodies and $\mathrm{C}$. Mark for editorial assistance. We used the equipment of the Core Facility "CHROMAS" (Biological Institute, SaintPetersburg State University). This work was supported by research grants 05-04-48252 from the Russian Foundation for Basic Research and 3.3/ 37483 from the Russian Ministry of Science and Education. The Department of Immunology and Oncology was founded and is supported by the Spanish Council for Scientific Research (CSIC) and by Pfizer.

\section{References}

Beenders B, Watrin E, Legagneux V, Kireev I, Bellini M (2003) Distribution of XCAP-E and XCAP-D2 in the Xenopus oocyte nucleus. Chromosome Res 11: 549-564.

Berezney R, Coffey DS (1974) Identification of nuclear protein matrix. Biochem Biophys Res Commun 60: 1410-1417.

Callan HG (1986) Lampbrush Chromosomes. Berlin: Springer.

Cohen-Fix O (2000) Sister chromatid separation: falling apart at the seams. Curr Biol 10: R816-R819.

Dobson MJ, Pearlman RE, Karaiskakis A, Spyropoulos B, Moens PB (1994) Synaptonemal complex proteins: occurrence, epitope mapping and chromosome disjunction. J Cell Sci 107: 2749-2760.

Doyle O, Corden JL, Murphy C, Gall JG (2002) The distribution of RNA polymerase II largest subunit (RPB1) in the Xenopus germinal vesicle. J Struct Biol 140: 154-166.

Dunn, MJ (1993) Gel Electrophoresis: Proteins. Oxford UK: BIOS Scientific Publishers.

Earnshaw WC, Halligan B, Cooke CA, Heck MMS, Liu LF (1985) Topoisomerase II is a structural component of mitotic chromosome scaffolds. J Cell Biol 100: 1706-1715.

Eijpe M, Heyting C, Gross B, Jessberger R (2000) Association of mammalian SMC1 and SMC3 proteins with meiotic chromosomes and synaptonemal complexes. J Cell Sci 113: 673-682. 
Firooznia A, Revenkova E, Jessberger R (2005) XXVII North American Testis Workshop: the function of SMC and other cohesin proteins in meiosis. $J$ Androl 26: 1-10.

Fisher D, Hock R, Sheer U (1993) DNA topoisomerase II is not detectable on lampbrush chromosomes but enriched in the amplified nucleoli of Xenopus oocytes. Exp Cell Res 209: 255-260.

Gaginskaya ER (1972) Nuclear structures in oocytes of adult birds. II. Protein bodies and the karyosphere. Tsitologiia 14: 568-578.

Gaginskaya ER, Gruzova MN (1969) Characteristics of oogenesis in the finch. Tsitologiia 11: 1241-1251.

Gall JG (1992) Organelle assembly and function in the amphibian germinal vesicle. Adv Dev Biol 1: 1-29.

Gall JG, Murphy C (1998) Assembly of lampbrush chromosomes from sperm chromatin. Mol Biol Cell 9: 733-747.

Gall JG, Wu Z, Murphy C, Gao H (2004) Structure in the amphibian germinal vesicle. Exp Cell Res 296: 28-34.

Gruber S, Haering CH, Nasmyth K (2003) Chromosomal cohesin forms a ring. Cell 112: 765-777.

Haering CH, Nasmyth K (2003) Building and breaking bridges between sister chromatids. Bioessays 25: 1178-1191.

Hagstrom KA, Meyer BJ (2003) Condensin and cohesin: more than chromosome compactor and glue. Natl Rev Genet 4: 520-534.

Hauf S, Roitinger E, Koch B, Dittrich CM, Mechtler K, Peters JM (2005) Dissociation of cohesin from chromosome arms and loss of arm cohesion during early mitosis depends on phosphorylation of SA2. PLoS Biol 3: e69.

Hock R, Carl M, Lieb B, Gebauer D, Scheer U (1996) A monoclonal antibody against DNA topoisomerase II labels the axial granules of Pleurodeles lampbrush chromosomes. Chromosoma 104: 358-366.

Jessberger R (2002) The many functions of SMC proteins in chromosome dynamics. Nat Rev Mol Cell Biol 3: 767-778.

Kouznetsova A, Novak I, Jessberger R, Hoog C (2005) SYCP2 and SYCP3 are required for cohesin core integrity at diplotene but not for centromere cohesion at the first meiotic division. $J$ Cell Sci 118: 2271-2278.

Krasikova A, Kulikova T, Saifitdinova A, Derjusheva S, Gaginskaya E (2004) Centromeric protein bodies on avian lampbrush chromosomes contain a protein detectable with an antibody against DNA topoisomerase II. Chromosoma 113: 316-323.

Kropotova EV, Gaginskaya ER (1984) Lampbrush chromosomes from Japanese quail oocytes. Tsitologiia 26: 1008-1014.

Lengronne A, Katou Y, Mori S et al. (2004) Cohesin relocation from sites of chromosomal loading to places of convergent transcription. Nature 430: 573-578.

Losada A, Yokochi T, Kobayashi R, Hirano T (2000) Identification and characterization of $\mathrm{SA} / \mathrm{Scc} 3 \mathrm{p}$ subunits in the Xenopus and human cohesion complexes. J Cell Biol 150: 405-416.

Macgregor, HC, Solovei, I, Mizuno, S, 1997. Lampbrush chromosomes as system for high resolution studies of meiotic chromosome structure. In: Abbondandolo A, Vig BK \& Roi R, eds. Chromosome Segregation and Aneuploidy. European Commission Joint Research Centre Publication, pp 172-183.

Meluh PB, Strunnikov AV (2002) Beyond the ABCs of CKC and SCC. Do centromeres orchestrate sister chromatid cohesion or vice versa? Eur J Biochem 269: 2300-2314.

Morgan GT (2002) Lampbrush chromosomes and associated bodies: new insights into principles of nuclear structure and function. Chromosome Res 10: 177-200.
Morrison C, Vagnarelli P, Sonoda E, Takeda S, Earnshaw WC (2003) Sister chromatid cohesion and genome stability in vertebrate cells. Biochem Soc Trans 31: 263-265.

Parra MT, Viera A, Gomez R et al. (2004) Involvement of the cohesin Rad21 and SCP3 in monopolar attachment of sister kinetochores during mouse meiosis I. J Cell Sci 117: 1221-1234.

Pezzi N, Prieto I, Kremer L et al. (2000) STAG3, a novel gene encoding a protein involved in meiotic chromosome pairing and location of STAG3-related genes flanking Williams-Beuren syndrome deletion. FASEB J 14: 581-592.

Prieto I, Suja JA, Pezzi N et al. (2001) Mammalian STAG3 is a cohesin specific to sister chromatid arms in meiosis I. Nat Cell Biol 3: 761-766.

Prieto I, Pezzi N, Buesa JM et al. (2002) STAG2 and Rad21 mammalian mitotic cohesins are implicated in meiosis. EMBO Rep 3: 543-550.

Prieto I, Tease C, Pezzi N et al. (2004) Cohesin component dynamics during meiotic prophase $\mathrm{I}$ in mammalian oocytes. Chromosome Res 12: 197-213.

Pyne CK, Loones M-T, Simon F, Zhou ZJ (1995) Immunocytochemical study of lampbrush chromosomes of the urodele Pleurodeles waltl: axial granules are recognized by the mitosis-specific monoclonal antibody MPM-2. Biol Cell 83: 191-200.

Revenkova E, Eijpe M, Heyting C, Gross B, Jessberger R (2001) Novel meiosis-specific isoform of mammalian SMC1. Mol Cell Biol 21: 6984-6998.

Ross KE, Cohen-Fix, O (2004) Cohesins slip sliding away. Nature 430: $520-521$.

Saifitdinova A, Derjusheva S, Krasikova A, Gaginskaya E (2003) Lampbrush chromosomes of the chaffinch (Fringilla coelebs L.). Chromosome Res 11: 93-113.

Schleiffer A, Kaitna S, Maurer-Stroh S, Glotzer M, Nasmyth K, Eisenhaber F (2003) Kleisins: a superfamily of bacterial and eukaryotic SMC protein partners. Mol Cell 11: 571-575.

Solari AJ (1981) Chromosomal axes during and after diplotene. Internatl Cell Biol 80-81: 178-186.

Solovei I, Gaginskaya E, Hutchison N, Macgregor H (1993) Avian sex chromosomes in the lampbrush form: the $\mathrm{ZW}$ lampbrush bivalent from six species of bird. Chromosome Res 1: 153-166.

Solovei IV, Gaginskaya ER, Macgregor HC (1994) The arrangement and transcription of telomere DNA sequences at the ends of lampbrush chromosomes of birds. Chromosome Res 2: 460-470.

Solovei IV, Joffe BI, Gaginskaya ER, Macgregor HC (1996) Transcription on lampbrush chromosomes of a centromerically localized highly repeated DNA in pigeon (Columba) relates to sequence arrangement. Chromosome Res 4: 588-603.

Tsvetkov AG, Gaginskaya ER (1983) The nuclear matrix of oocytes of the chaffinch (Fringilla coelebs L.). Tsitologiia 25: 649-654.

Watanabe Y, Nurse P (1999) Cohesin Rec8 is required for reductional chromosome segregation at meiosis. Nature 400: 461-464.

Xu H, Beasley M, Verschoor S, Inselman A, Handel MA, McKay MJ (2004) A new role for the mitotic RAD21/SCC1 cohesin in meiotic chromosome cohesion and segregation in the mouse. EMBO Rep 5: 378-384. 